\title{
cAMP Agonist Forskolin Disrupts Mitochondrial Metabolism and Induces Senescence in Human Mesenchymal Cells
}

Rongjia Zhu

Chinese Academy of Medical Sciences \& Peking Union Medical College

Qiaoling Wang

Chinese Academy of Medical Sciences \& Peking Union Medical College

Xiaodong Su

Beijing Tiantan Hospital

Kan Yin

Qingdao University

Robert Chunhua Zhao ( $\nabla$ zhaochunhua@ibms.pumc.edu.cn )

Chinese Academy of Medical Sciences and Peking Union Medical College https://orcid.org/0000-00015740-4506

\section{Research Article}

Keywords: Mesenchymal stem cells, stem cell therapy, energy metabolism dysfunction, senescence, longterm culture

Posted Date: June 25th, 2021

DOI: https://doi.org/10.21203/rs.3.rs-634834/v1

License: (c) (i) This work is licensed under a Creative Commons Attribution 4.0 International License. Read Full License 


\section{Abstract}

Backgroud: Adult-derived mesenchymal stem cells (MSCs) can be used in therapies for the treatment of various diseases. However, MSCs derived from aging tissues or long-term MSC cultures could have diminished therapeutic effects compared with MSCs derived from younger tissues, but the underlying mechanism has not been completely established. Dysfunction of energy metabolism is one of the main mechanisms underlying cell senescence. Although cyclic adenosine monophosphate (cAMP) is known to inhibit cell division and proliferation in vitro, its impact on MSC senescence has not been described.

Methods: In this study, we used forskolin, an adenylate cyclase agonist and cAMP inducer, to disrupt metabolism in human adipose-derived MSCs and investigate the effects of metabolic dysfunction on MSC senescence. CAMP and ATP levels were identified by its ELISA kits. The identification of MSCs senescence was assessed by MTS assay, cell cycle tests, $\beta$-galactosidase staining, and genes evaluation through qRT-PCR and Western blotting. Functions of the forskolin treated-MSCs were evaluated by oil Red $O$ staining and alkaline phosphatase staining, as well as mRNA tests and wound healing assay. Mitochondrial morphology and function tests were performed by transmission electron microscopy and oxygen consumption analysis, mitochondrial superoxide assay, quantification of nitric oxide.

Results: Treatment of human MSCs with forskolin resulted in senescence phenotypes, including reduced proliferation, cell-cycle arrest, and enhanced expression of the cell aging markers P16 and P21.

Furthermore, the senescent MSCs exhibited increased adipogenesis capacity and decreased osteogenesis capacity as well as a senescence-associated secretory phenotype characterized by increased expression of several inflammatory factors. Forskolin-associated MSC senescence was mainly caused by oxidative stress-induced disruption of mitochondrial metabolism, and the senescent MSCs had high levels of reactive oxygen species and reduced sirtuin gene expression. Lastly, we found that CAMP inhibitor SQ22536 protects MSCs from forskolin-induced senescence and senescence-related-inflammatoryphenotype (SASP).

Conclusions: Our results indicate that forskolin can cause senescence of human MSCs through oxidative stress-induced mitochondrial metabolic dysfunction.

\section{Background}

Adult mesenchymal stem cells (MSCs) have multiple applications for the treatment of various diseases, mainly owing to their powerful immunomodulatory and regenerative features, paracrine effects, multilineage differentiation potential, and migration ability. Because the human body has relatively few MSCs, MSCs are typically expanded in vitro to obtain a sufficient amount of cells for clinical applications. During culture in vitro, MSCs may undergo senescence owing to their limited capacity for continuous cell division[1-5]. Aged MSCs generally have relatively reduced efficacy compared with younger MSCs in various disease models. And the donor's age and/or health status may limit the therapeutic potential of the harvested MSCs[6, 7]. This can be partly attributable to the fact that senescent MSCs may produce 
inflammatory cytokines, chemokines, and/or extracellular matrix-remodeling factors that can disrupt tissue structure and function.

Cellular metabolic dysfunction may reflect cellular senescence, tissue aging, and several aging-related diseases such as Alzheimer's disease, Parkinson's disease, stroke, and osteoarthritis[8-12]. The cyclic ADP-ribose hydrolase $\mathrm{CD} 38$ regulates metabolism during aging by modulating cellular NAD ${ }^{+}$level and the activity of the histone deacetylase sirtuin 3 (SIRT3)[13]. Agonists of the nuclear receptor PPARy have shown therapeutic potential for certain age-related brain disorders[14].

Cyclic adenosine monophosphate (CAMP) is a second-messenger molecule that plays important roles in various cellular physiological and pathological processes including metabolism, cell differentiation, and inflammation[15]. Forskolin, a natural compound produced in the roots of the Indian plant Coleus forskohlii, directly activates adenylate cyclase, which generates CAMP from ATP, thereby increasing the intracellular concentration of cAMP[16]. As an adenylate cyclase agonist, forskolin inhibits cell proliferation, motility, colony formation, and migration and promotes apoptosis and improves the sensitivity of various types of tumor cells to chemotherapeutic drugs; therefore, forskolin has applications for the treatment of several cancers[17-21].

MSCs are present in almost every tissue and are essential for tissue longevity. Because cAMP is generated from ATP, we carried out experiments to determine the effects of the cAMP agonist forskolin on MSC senescence and studied the underlying mechanism.

\section{Methods}

\section{Preparation and treatment of MSCs}

MSCs were isolated from discarded adipose tissue removed from healthy patients undergoing liposuction surgery, as described [22]. Informed consent was obtained from all patients. The procedure was approved by the Ethics Committee of the Chinese Academy of Medical Sciences and Peking Union Medical College. The isolated cells were seeded in T75 flasks containing $12 \mathrm{~mL}$ culture medium and incubated under humidified conditions at $37^{\circ} \mathrm{C}$ and $5 \% \mathrm{CO}_{2}$. MSCs were continuously cultured to the third passage and then used for experiments. Cells were either left untreated or treated for $24 \mathrm{~h}$ with forskolin $(60 \mu \mathrm{M}$, Target Mol, Boston, MA, USA). Pyrrolidine dithiocarbamic acid (PDTC, Beyotime, Shanghai, China) $100 \mu \mathrm{M}$ or

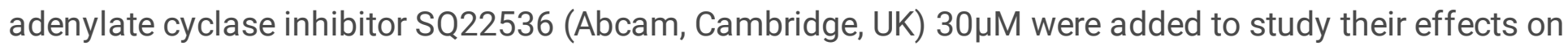
MSCs senescence.

\section{MTS assay}

An MTS (3-(4,5-dimethylthiazol-2-yl)-5-(3-carboxymethoxyphenyl)-2-(4-sulfophenyl)-2H-tetrazolium) assay kit (Sigma-Aldrich, St. Louis, MO, USA) was used to assess cell viability. MSCs were seeded into five 96well plates at a density of $2 \times 10^{3}$ cells per well, with each plate being cultured for $0,24,48,72$, or $96 \mathrm{~h}$. One hour before each time point, $20 \mu \mathrm{L}$ MTS reagent was added into each well of the 96 -well plate 
containing $100 \mu \mathrm{L}$ culture medium. After incubation at $37^{\circ} \mathrm{C}$ for $1 \mathrm{~h}$, absorbance at $490 \mathrm{~nm}$ was measured using a 96-well plate reader (Bio-Rad, Hercules, CA, USA).

\section{Assessment of senescence using $\beta$-galactosidase staining}

A senescence $\beta$-Galactosidase staining kit (Beyotime) was used for staining senescent cells based on the upregulation of senescence-associated $\beta$-galactosidase activity during senescence. Culture medium was aspirated from 6-well plates, and the wells were washed once with phosphate-buffered saline (PBS). The cells were then fixed at room temperature for 15 min with $1 \mathrm{~mL} \beta$-galactosidase staining fixative solution (from the kit) per well and then washed three times with PBS. Finally, $1 \mathrm{~mL}$ of working solution of the stain was added to each well, and the plates were incubated overnight at $37^{\circ} \mathrm{C}$ in a $\mathrm{CO}_{2}$-free incubator. The cells were then observed and counted under an optical microscope.

\section{Quantification of CAMP}

The intracellular concentration of cAMP was determined with a cAMP ELISA kit (Elabscience, Wuhan, China), which uses a competitive ELISA method. Briefly, wells of a microtiter plate were coated with cAMP (the antigen), and the cAMP present in each cell extract or standard solution was allowed to compete with the immobilized cAMP for binding to a biotin-labeled monoclonal antibody against cAMP. Unbound components were then washed away. Horseradish peroxidase (HRP) conjugated avidin was then added, resulting in the formation of an immune complex; the solution in each well was aspirated, and the plates were washed three times with PBS. The chromogenic substrate TMB was then added, which turned the solution blue upon catalysis by HRP and then yellow after the stop solution was added. Finally, absorbance at $450 \mathrm{~nm}$ was measured with a microplate reader, and the cAMP concentration in each sample was calculated based on a standard curve.

\section{Cell-cycle analysis}

Cell-cycle phase was determined by flow cytometry using propidium iodide staining. MSCs were seeded into 6-well plates at a density of $2 \times 10^{5}$ cells per well and then treated with DMSO (vehicle control) or forskolin ( $60 \mu \mathrm{M}$ final concentration) for $24 \mathrm{~h}$. Cells were then harvested and fixed in $1 \mathrm{~mL}$ of $75 \%$ ethanol at $4^{\circ} \mathrm{C}$ for $24 \mathrm{~h}$. The fixed cells were then washed twice and resuspended in $50 \mu \mathrm{L}$ PBS, followed by addition of $5 \mu \mathrm{L}$ RNase $\mathrm{A}(10 \mathrm{mg} / \mathrm{mL})$ and incubation at $37^{\circ} \mathrm{C}$ for $30 \mathrm{~min}$. Next, $450 \mu \mathrm{L}$ PBS with $10 \mu \mathrm{L}$ propidium iodide $(1 \mathrm{mg} / \mathrm{mL})$ was added, and the samples were incubated at $4^{\circ} \mathrm{C}$ in the dark for 30 min. Cells were detected with a flow cytometer (Accuri ${ }^{\mathrm{TM}} \mathrm{C6}$, Becton Dickinson, Franklin Lakes, NJ, USA), and the data were analyzed using CFlow Plus software (Becton Dickinson) and ModFit (Verity Software House, Topsham, ME, USA).

\section{Transmission electron microscopy}

MSCs were treated with DMSO (control) or forskolin and then fixed in $2 \%$ glutaraldehyde solution in $0.1 \mathrm{M}$ cacodylate buffer for $24 \mathrm{~h}$, post-fixed in $1 \%$ osmium tetroxide $\left(\mathrm{OsO}_{4}\right)$ for $1 \mathrm{~h}$, and dehydrated in sequential dilutions of acetone (25\%, 50\%, 75\%, and 100\% twice) before impregnation in resin of increasing concentration (25\%, 50\%, 75\%, and 100\% three times) in acetone over a $24 \mathrm{~h}$ period. Each sample was 
subjected to transmission electron microscopy, and mitochondrial morphology was assessed and imaged at magnifications of $8000 x$ and $10,000 \times$.

\section{Measurement of intracellular ATP content}

Intracellular ATP content was measured with an ATP assay kit (Beyotime). Briefly, cells were lysed and then centrifuged at $12,000 \cdot g$ for $10 \mathrm{~min}$ at $4^{\circ} \mathrm{C}$. The supernatant was removed and mixed with dilution buffer from the kit, after which the relative light units were measured using a luminometer. A standard curve was prepared for each round of assays to calculate ATP content.

\section{Measurement of intracellular reactive oxygen species (ROS)}

Mitochondrial ROS formation was assayed using 2,7-dichlorofluorescein diacetate, a fluorescent probe, as part of a ROS assay kit (Beyotime) with a slight procedural modification. Briefly, MSCs were incubated with the probe $(10 \mu \mathrm{M})$ dissolved in serum-free DMEM at $37^{\circ} \mathrm{C}$ for $20 \mathrm{~min}$. Fluorescence was measured via excitation at $488 \mathrm{~nm}$ and emission at $525 \mathrm{~nm}$ with a FACSCalibur ${ }^{\mathrm{TM}}$ flow cytometer (BD Biosciences, San Jose, CA, USA).

\section{Mitochondrial superoxide assay}

Mitochondrial superoxide (SOX) formation was assayed with the MitoSOX Red kit (Yesen, Shanghai, China). First, $13 \mu \mathrm{L}$ DMSO and $50 \mu \mathrm{g}$ MitoSOX Red (the probe; Yesen) were mixed well to prepare a $5 \mathrm{mM}$ MitoSOX Red working solution. Next, a $5 \mu \mathrm{M}$ probe working solution was prepared by diluting the MitoSOX Red working solution with serum-free culture medium. The cells were washed twice with PBS and resuspended with $2 \mathrm{~mL}$ of the probe working solution. After incubation at $37^{\circ} \mathrm{C}$ for $10 \mathrm{~min}$ in the dark, the cells were washed three times with PBS. Fluorescence was measured with a FACSCalibur excitation $488 \mathrm{~nm}$, emission $525 \mathrm{~nm}$.

\section{Quantification of nitric oxide}

Dilute DAF-FM DA (Beyotime)with the DAF-FM DA diluent provided in this kit to a final concentration of 5 $\mu \mathrm{mol} / \mathrm{L}$. After the cells were collected, resuspend the cells with diluted DAF-FM DA for 20 min at $37^{\circ} \mathrm{C}$, then washed with PBS to remove excess probe. Fluorescence was measured with a FACSCalibur excitation $495 \mathrm{~nm}$, emission max $515 \mathrm{~nm}$.

\section{Oxygen consumption analysis}

The Seahorse XF24 Extracellular Flux Analyzer (Seahorse Bioscience, North Billerica, MA, USA) was used to measure oxygen consumption in MSCs. Forskolin-treated and control MSCs were plated onto 24 well XF cell culture microplates at a density of 50,000 cells/well in $250 \mu \mathrm{L}$ culture medium on the day before analysis. XF assay medium was supplemented with $250 \mathrm{mM}$ glucose and $2 \mathrm{mM}$ sodium pyruvate. Hydrate a sensor cartridge in XF Calibrant at $37^{\circ} \mathrm{C}$ in a non-CO2 incubator overnight. After basal respiration measurements, cells were treated sequentially with oligomycin $(1 \mathrm{mM})$ to measure the nonphosphorylating OCR, carbonyl cyanide-4-(trifluoromethoxy) phenylhydrazone $(0.5 \mathrm{mM})$ to determine maximal OCR, and rotenone and antimycin A mixture $(0.5 \mathrm{mM})$ to measure the residual mitochondrial 
$\mathrm{OCR}$, and changes in respiration were recorded. Use the default software settings analyzed in Wave or XF software.

\section{Wound healing assay}

Wound healing assays were performed using Culture-Insert two-well inserts (Ibidi, Munich, Bavaria, Germany). Briefly, the inserts were placed in 6-well plates prior to seeding the cells and were removed after the cells formed a confluent layer. The remaining medium was replaced with fresh serum-free medium, and images were acquired at the indicated time points. After $24 \mathrm{~h}$, the distances traveled by cells were measured in forskolin-treated and non-treated MSCs.

\section{Alkaline phosphatase staining}

Alkaline phosphatase (ALP) staining was performed using an ALP staining kit (Institute of Hematology and Blood Diseases Hospital, Chinese Academy of Medical Sciences, Tianjin, China) The cells were incubated with this staining solution for $1 \mathrm{~h}$ at $37^{\circ} \mathrm{C}$ in a $\mathrm{CO}_{2}$-free incubator, washed with PBS, and images were acquired under a light microscope.

\section{Oil red 0 staining}

Cells plated in 24-well plates were washed twice with PBS, fixed with $4 \%$ paraformaldehyde for 10 min, and stained with filtered oil red 0 solution (stock solution: $1 \mathrm{mg} / \mathrm{mL}$ in isopropanol; working solution: $60 \%$ oil red $\mathrm{O}$ stock solution mixed with $40 \%$ distilled water) at $37^{\circ} \mathrm{C}$ for $30 \mathrm{~min}$. The cells were rinsed with distilled water to remove unbound dye and then observed and photographed under a light microscope.

\section{RNA isolation and quantitative real-time polymerase chain reaction (qPCR) analysis}

Total RNA was extracted from cultured cells with TRIzol reagent (Invitrogen, Carlsbad, CA, USA), and the RNA was quantified with a Model ND1000 spectrophotometer from NanoDrop Technologies (Wilmington, DE, USA). Reverse transcription was performed using a Reverse Transcription kit (Takara, Tokyo, Japan). qPCR was performed with SYBR premix Ex Taq (Takara) with the Step One Plus Real-Time PCR Detection System (Applied Biosystems, Foster City, CA, USA). The relative expression of mRNA was evaluated by the $2^{-\triangle \Delta C t}$ method and normalized to the expression of GAPDH. Supplementary Table 1 lists the primers used in this study.

\section{Western blotting}

Cells were harvested in RIPA lysis buffer (Beyotime) supplemented with $1 \mathrm{mM}$ phenylmethanesulfonyl fluoride. Total protein was quantified using a BCA Protein Assay kit (Beyotime). Cell extracts (20 $\mu \mathrm{g}$ protein) were subjected to SDS-PAGE (10\% acrylamide gels) and then transferred to a $0.45-\mu \mathrm{m}$ polyvinylidene difluoride membrane (Millipore, Boston, MA, USA). After blocking with $5 \%$ fat-free milk in Tris-buffered saline Tween-20 (TBST) for $1 \mathrm{~h}$ at room temperature, each membrane was incubated with a specific primary antibody at $4^{\circ} \mathrm{C}$ overnight. The next day, each membrane was incubated with a HRPconjugated secondary antibody for $1 \mathrm{~h}$ at room temperature. Immunopositive bands were visualized with 
HRP substrate (Proteintech, Rosemont, IL, USA) and quantified using an Image Quant LAS 4000 mini imaging system (GE Healthcare, Chicago, IL, USA). GAPDH was used as an internal control. Primary antibodies (rabbit IgG, from Proteintech) were used that targeted the following proteins: P16 (diluted 1:1000), P21 (1:1000), P53 (1:2000), and lamin B1 (1:2000).

\section{Statistical analysis}

Data were analyzed using Prism 8 software (GraphPad Software Inc. USA). The Student's t-test was used for comparisons between groups. $P$-values of $<0.05$ were considered to reflect statistically significant differences between data. Error bars indicate standard deviation.

\section{Results}

\section{Forskolin increases cAMP level and reduced ATP level in MSCs}

To study the effects of forskolin on MSCs, we first treated MSCs with $60 \mathrm{mM}$ forskolin and monitored changes in CAMP and ATP levels. CAMP production in forskolin-treated MSCs (12 or $24 \mathrm{~h}$ ) was 3-fold greater than that in control MSCs, and this increase subsided after $48 \mathrm{~h}$ (Fig. 1a). Forskolin significantly reduced intracellular ATP level after $24 \mathrm{~h}$ (data not shown) and $48 \mathrm{~h}$ (Fig. 1b). Thus, forskolin affected cAMP and ATP levels in MSCs.

\section{Forskolin leads to a senescence phenotype in MSCs}

Certain cAMP-elevating agents and cAMP analogs can inhibit cell proliferation[23]. We found that a 24-h incubation with forskolin significantly decreased MSC proliferation compared with control MSCs (Fig. 2a). Cell-cycle analysis of forskolin-treated MSCs revealed a significant arrest at the S and G2 + M phases (Fig. 2b). With regard to MSC senescence, after one day of treatment with forskolin, $\beta$ galactosidase staining was observed in $82.33 \pm 4.04 \%$ of forskolin-treated MSCs and $7.77 \pm 2.08 \%$ of control cells, indicating that a forskolin-induced increase in cAMP level could induce senescence (Fig. 2c). This conclusion was supported by the forskolin-induced upregulation of molecular markers associated with cell aging, namely P16 and P21, and downregulation of lamin B1 (Fig. 2d, e).

\section{Forskolin alters the differentiation capacity of MSCs}

We next studied the immunophenotype of the MSCs after forskolin treatment. Both the forskolin-treated and control MSCs expressed CD44 and CD29 but not HLA-DR, CD45, and CD31 (Fig. 3a). Oil red O staining is commonly used to detect lipogenesis. And Alkaline phosphatase staining is commonly used to detect osteogenesis. Forskolin-treated MSCs showed strong Oil red 0 staining yet weak ALP staining (Fig. 3b), and the early adipocyte genes $\angle P L, C / E B P a$, and $P P A R Y$ were significantly upregulated compared with the control groups (Fig. 3c), and the osteogenesis genes $A L P, R U N X 2$, and OPN were 
downregulated (Fig. 3d). Thus, forskolin-treated MSCs had a normal immunophenotype but greater adipogenic capacity and lesser osteogenic capacity than the control MSCs.

\section{Forskolin induces senescence-related transcriptional reprogramming}

To determine the mechanism underlying the effects of forskolin on MSCs, we exposed MSCs to $60 \mu \mathrm{M}$ forskolin for $24 \mathrm{~h}$ and identified 3154 upregulated and 2731 downregulated genes, indicating that forskolin triggered global transcriptional reprogramming in MSCs (Fig. 4a). Gene Ontology enrichment of differentially expressed genes revealed cAMP catabolic processes and cAMP-mediated signaling as two of the top four activated processes (Fig. 4b), and thus forskolin indeed targeted the expected pathways in our experiment. Two other activated processes, namely apoptosis and oxidative stress response, also supported our result that forskolin triggered MSC senescence (Fig. 4b). Several senescence- and differentiation-related processes also differed between forskolin-treated and control MSCs, such as osteoblast differentiation, cell proliferation, and cell-cycle processes (Fig. 4b). Moreover, several of the top 15 enriched KEGG pathways were related to senescence (Fig. c). Interestingly, certain inflammationrelated pathways differed significantly between the two groups of cells, such as the NF-KB signaling and TNF signaling pathways (Fig. 4c).

Because cell-cell adhesion was the most significantly downregulated Gene Ontology process (Fig. 4b), we analyzed the effect of forskolin on MSC migration. In wound healing assays, the average distance traveled by cells in the forskolin group was significantly less than that of control MSCs (Fig. 4d). Senescent cells typically exhibit characteristics of producing inflammatory factors, and thus they are often referred to as having a senescence-related secretory phenotype (SASP)[24, 25]. qPCR was thus performed to assess whether forskolin treatment resulted in a SASP. MSCs treated with forskolin expressed lower levels of the pluripotent genes encoding Nanog and Sox2 (Fig. 4e) but much higher levels of several proinflammatory factors, including IL-6, IL-8, IL-1 control MSCs. Further, the longevity genes encoding SIRT1, 2, 3, and 6 were downregulated in forskolintreated cells, confirming cellular aging (Fig. 4f). Together, these results suggested that forskolin caused global transcriptional reprogramming in MSCs that contributed to senescence-related cellular functions.

\section{Forskolin disrupts mitochondrial metabolism in MSCs}

We next studied mitochondrial function, which is key for regulating cell viability, in forskolin-treated MSCs. Forskolin-treated MSCs had atypical mitochondria that were untethered, swollen, yet small, compared with mitochondria in control MSCs. (Fig. 5a). Mitochondrial metabolism-associated functions were downregulated in forskolin-treated MSCs, including the processes of ATP production, basal and maximal respiration, and the proton gradient (Fig. 5b-d). However, there was no statistically significant difference in coupling efficiency and non-mitochondrial oxygen consumption between the two groups (Fig. 5e). Forskolin did not affect the total number of mitochondria (Fig. 5f) but increased the production of ROS and SOX (Fig. 5g, h), NO (Fig. 5i), and calcium flux (Fig. 5j). Moreover, forskolin-treated MSCs 
exhibited upregulated expression of the NO-induced genes $\mathrm{HO}^{-1}$ and $\mathrm{FOXO}-1$ (Fig. 5k), the latter being a ROS-regulated gene expressed at higher levels in older muscle than in younger muscle[26]. The expression of BACH-1, an inhibitor of HO-1, was also activated in forskolin-treated MSCs (Fig. 5k). These results suggested that mitochondria of forskolin-treated senescent MSCs were impaired with respect to both morphology and metabolism."

\section{An adenylate cyclase inhibitor blocks forskolin-induced MSC senescence}

The NF-KB pathway is essential for SASPs [27], and thus we treated MSCs with the NF-KB inhibitor pyrrolidine dithiocarbamic acid (PDTC) $100 \mu \mathrm{M}$ to determine whether NF-kB inhibition could rescue forskolin-induced senescence. To our surprise, the cAMP content increased after $24 \mathrm{~h}$ of PDTC treatment (Fig. 6a). Although the levels of IL-6 and IL-8 mRNAs decreased, IL-1 $\beta$ expression was increased (Fig. 6b). Moreover, the senescence-related gene $P 16$ was downregulated whereas $P 21$ was upregulated (Fig. $6 \mathrm{c}$ ).

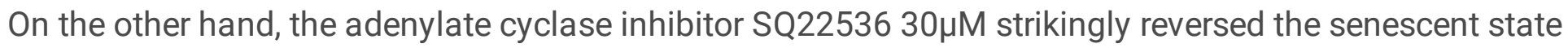
of forskolin-treated MSCs, exemplified by the decreased expression of each of P16, P21, and the inflammatory factors IL-8, IL-1 $\beta$, and TNF-a (Fig. 6d). Thus, the adenylate cyclase inhibitor SQ22536, but not PDTC, could effectively reverse forskolin-induced senescence.

\section{Discussion}

MSCs have shown potential in clinical trials for the treatment of several human diseases. However, preventing MSC senescence during in vitro culture remains a challenge for clinical applications of MSCs. Thus, deciphering the critical factors that contribute to MSC senescence is of great importance for developing effective strategies to prolong the viability of MSCs destined for clinical use. In this study, we treated MSCs with forskolin in vitro and demonstrated that forskolin significantly induces MSC senescence and abnormal differentiation. Forskolin triggered global transcriptional reprogramming of MSCs, thereby reducing their viability, disrupting mitochondrial metabolism, and producing other senescence-related phenotypes.

An increasing body of evidence has shown that metabolic changes within individual cells affect cell-fate decisions including entry into an apoptosis or senescence program[28-32]. cAMP is generated in both the mitochondria and cytoplasm by adenylate cyclase in response to metabolically generated carbon dioxide [33], and cAMP signaling is important for regulating mitochondrial biogenesis. The natural cAMPelevating compound forskolin raises intracellular cAMP levels by activating adenylate cyclase, which generates cAMP from ATP. Because forskolin alters cellular energy metabolism, it has been proposed as an effective anticancer agent for several cancer types[34]. However, the role of forskolin in MSC senescence remains unknown. We found that forskolin-induced senescence of MSCs resulted in abnormal mitochondrial morphology and impaired metabolism-associated functions, particularly increased production of both ROS and NO and increased $\mathrm{Ca}^{2+}$ flux. Surprisingly, although both RNA-seq and qPCR data revealed that forskolin-treated MSCs had a SASP, the NF-KB inhibitor PDTC did not 
prevent forskolin-induced MSC senescence (rather, it only partially reduced the expression of genes encoding proinflammatory cytokines). These results indicate that the NF-KB pathway is not the primary mediator of forskolin-induced MSC senescence and that the observed SASP is a consequence of events occurring downstream of senescence. One the other hand, SQ22536, an adenylyl cyclase inhibitor that inhibits forskolin-induced cAMP elevation[35], remarkably reversed forskolin-induced MSC senescence.

Research has shown that forskolin at $4 \mu \mathrm{M}$ promotes mitochondrial biogenesis and at $4 \mu \mathrm{M}$ upregulates fatty-acid oxidation[36]. In contrast, use of a higher dose $(60 \mu \mathrm{M})$ in our experiments revealed that forskolin alters mitochondrial morphology and function in MSCs and triggers their senescence (Fig. 7). This phenomenon suggests that forskolin may have contrasting effects in different contexts or at different doses. For example, eye drops containing forskolin have passed all clinical trial phases, and 10 $\mathrm{mg} /$ day of forskolin could prevent asthma in children and adults with mild/moderate persistent asthma via an increase in FEV1 and a decrease frequency in asthmatic attacks[37-39]. Moreover, our study may provide probable references of forskolin for clinical use, such as cumulative dose repeatedly and drug resistance mechanism. Additionally, forskolin has also been proposed for anticancer treatments. Thus, our results that forskolin can induce MSC senescence improves our knowledge of the role forskolin plays in cellular energy metabolism yet raises caution regarding its clinical applications.

Preventing senescence has been a long-standing goal of developmental biologists, and MSCs have proved promising for anti-aging strategies countering the aging process. Our study reveals that forskolin can potently induce MSC senescence. Moreover, an adenylyl cyclase inhibitor could potentially be an effective means of countering forskolin-induced MSC senescence, thus providing a basis for further research toward the goal of optimizing cAMP signaling to minimize the impact of senescence on MSCs. However, our study has certain limitations that require further investigation. For example, although our results provide solid evidence that forskolin induces MSC senescence via transcriptional reprogramming and disrupting mitochondrial metabolism, any potential interactions between these two aspects remains unclear. Because mitochondrial metabolism is key for controlling cell fate, further studies to reveal subtle changes in both the nuclear and mitochondrial transcriptomes may suggest practical means for preventing forskolin-induced senescence in vitro. Furthermore, the opposing effects of low-dose and highdose forskolin-as demonstrated in our current study and previous studies-raises the necessity to perform in vivo studies to further investigate the dose-related effects of forskolin on mitochondrial metabolism and senescence.

\section{Conclusions}

We found that forskolin can cause senescence of human MSCs with distinctive differentiation abilities and secretory functions through oxidative stress-induced mitochondrial metabolic dysfunction. These results suggest the mechanism of senescence induced by cAMP metabolic disorders in MSC, and thus the results provide a basis for developing strategies for improving the quality and efficacy of cultured MSCs for clinical use. 


\section{Abbreviations}

MSCs

Mesenchymal stem cells

cAMP

cyclic adenosine monophosphate

SASP

senescence-related-inflammatory-phenotype

PDTC

Pyrrolidine dithiocarbamic acid

ALP

Alkaline phosphatase

GAPDH

Glyceraldehyde-3-phodphate dehydrogenase

\section{Declarations}

\section{Ethics approval and consent to participate}

Human adipose tissues were medical waste obtained from patients undergoing liposuction. The samples are only used for scientific research. The collection of samples does not cause additional harm to the patients, and does not infringe their privacy. The informed consent was exempted, which was approved by the Ethics Committee at the Chinese Academy of Medical Sciences and Peking Union Medical College.

\section{Consent for publication}

Not applicable.

\section{Availability of data and materials}

All data generated or analyzed during this study are included in this published article.

\section{Competing interests}

The authors declare that they have no competing interests.

\section{Funding}

This work was supported by the National Key Research and Development Program of China (2016YFA0101000, 2016YFA0101003, 2018YFA0109800), CAMS Innovation Fund for Medical Sciences (2017-I2M-3-007), the 111 Project (B18007), and the National Natural Science Foundation of China (81672313, 81700782, 81971324, 81972523, 81771349). 


\section{Authors' contributions}

The study was designed by Rongjia Zhu and Robert Chunhua Zhao. Qiaoling Wang carried out the experiments, performed the statistical analyses. Xiaodong Su and Kan Yin helped with some of the experiments. All authors have read and approved the final manuscript.

\section{Acknowledgements}

Not applicable.

\section{References}

1. Bajek A, Czerwinski M, Olkowska J, Gurtowska N, Kloskowski T, Drewa T. Does aging of mesenchymal stem cells limit their potential application in clinical practice? Aging Clin Exp Res. 2012;24(5):404-11.

2. Banfi A, Muraglia A, Dozin B, Mastrogiacomo M, Cancedda R, Quarto R. Proliferation kinetics and differentiation potential of ex vivo expanded human bone marrow stromal cells: Implications for their use in cell therapy. Exp Hematol. 2000;28(6):707-15.

3. Lepperdinger G. Inflammation and mesenchymal stem cell aging. Curr Opin Immunol. 2011;23(4):518-24.

4. von Bahr L, Sundberg B, Lonnies L, Sander B, Karbach H, Hagglund H, Ljungman P, Gustafsson B, Karlsson H, Le Blanc K, et al. Long-term complications, immunologic effects, and role of passage for outcome in mesenchymal stromal cell therapy. Biol Blood Marrow Transplant. 2012;18(4):557-64.

5. Wang Y, Han ZB, Song YP, Han ZC. Safety of mesenchymal stem cells for clinical application. Stem Cells Int. 2012;2012:652034.

6. Duggal S, Brinchmann JE. Importance of serum source for the in vitro replicative senescence of human bone marrow derived mesenchymal stem cells. J Cell Physiol. 2011;226(11):2908-15.

7. Sotiropoulou PA, Perez SA, Salagianni M, Baxevanis CN, Papamichail M. Characterization of the optimal culture conditions for clinical scale production of human mesenchymal stem cells. Stem Cells. 2006;24(2):462-71.

8. Kapogiannis D, Mattson MP. Disrupted energy metabolism and neuronal circuit dysfunction in cognitive impairment and Alzheimer's disease. Lancet Neurol. 2011;10(2):187-98.

9. Lucke-Wold BP, Turner RC, Lucke-Wold AN, Rosen CL, Huber JD. Age and the metabolic syndrome as risk factors for ischemic stroke: improving preclinical models of ischemic stroke. Yale J Biol Med. 2012;85(4):523-39.

10. O'Donnell C, Migliore E, Grandi FC, Koltsov J, Lingampalli N, Cisar C, Indelli PF, Sebastiano V, Robinson WH, Bhutani N, et al. Platelet-Rich Plasma (PRP) From Older Males With Knee Osteoarthritis Depresses Chondrocyte Metabolism and Upregulates Inflammation. J Orthop Res. 2019;37(8):1760-70. 
11. Ryan BJ, Hoek S, Fon EA, Wade-Martins R. Mitochondrial dysfunction and mitophagy in Parkinson's: from familial to sporadic disease. Trends Biochem Sci. 2015;40(4):200-10.

12. Yin F, Sancheti $H$, Patil I, Cadenas E. Energy metabolism and inflammation in brain aging and Alzheimer's disease. Free Radic Biol Med. 2016;100:108-22.

13. Camacho-Pereira J, Tarrago MG, Chini CCS, Nin V, Escande C, Warner GM, Puranik AS, Schoon RA, Reid JM, Galina A, et al. CD38 Dictates Age-Related NAD Decline and Mitochondrial Dysfunction through an SIRT3-Dependent Mechanism. Cell Metab. 2016;23(6):1127-39.

14. Ulrich-Lai YM, Ryan KK. PPARgamma and stress: implications for aging. Exp Gerontol. 2013;48(7):671-6.

15. Sutherland EW, Rall TW. Fractionation and characterization of a cyclic adenine ribonucleotide formed by tissue particles. J Biol Chem. 1958;232(2):1077-91.

16. Dessauer CW, Watts VJ, Ostrom RS, Conti M, Dove S, Seifert R. International Union of Basic and Clinical Pharmacology. Cl. Structures and Small Molecule Modulators of Mammalian Adenylyl Cyclases. Pharmacol Rev. 2017;69(2):93-139.

17. Burdyga A, Conant A, Haynes L, Zhang J, Jalink K, Sutton R, Neoptolemos J, Costello E, Tepikin A. CAMP inhibits migration, ruffling and paxillin accumulation in focal adhesions of pancreatic ductal adenocarcinoma cells: effects of PKA and EPAC. Biochim Biophys Acta. 2013;1833(12):2664-72.

18. Dong H, Claffey KP, Brocke S, Epstein PM. Inhibition of breast cancer cell migration by activation of cAMP signaling. Breast Cancer Res Treat. 2015;152(1):17-28.

19. Follin-Arbelet V, Misund K, Naderi EH, Ugland H, Sundan A, Blomhoff HK. The natural compound forskolin synergizes with dexamethasone to induce cell death in myeloma cells via BIM. Sci Rep. 2015;5:13001.

20. Naviglio S, Di Gesto D, Illiano F, Chiosi E, Giordano A, Illiano G, Spina A. Leptin potentiates antiproliferative action of cAMP elevation via protein kinase A down-regulation in breast cancer cells. J Cell Physiol. 2010;225(3):801-9.

21. Wang H, Lou C, Ma N. Forskolin exerts anticancer roles in non-Hodgkin's lymphomas via regulating Axin/beta-catenin signaling pathway. Cancer Manag Res. 2019;11:1685-96.

22. Cao Y, Sun Z, Liao L, Meng Y, Han Q, Zhao RC. Human adipose tissue-derived stem cells differentiate into endothelial cells in vitro and improve postnatal neovascularization in vivo. Biochem Biophys Res Commun. 2005;332(2):370-9.

23. McEwan DG, Brunton VG, Baillie GS, Leslie NR, Houslay MD, Frame MC. Chemoresistant KM12C colon cancer cells are addicted to low cyclic AMP levels in a phosphodiesterase 4-regulated compartment via effects on phosphoinositide 3-kinase. Cancer Res. 2007;67(11):5248-57.

24. Coppe JP, Patil CK, Rodier F, Sun Y, Munoz DP, Goldstein J, Nelson PS, Desprez PY, Campisi J. Senescence-associated secretory phenotypes reveal cell-nonautonomous functions of oncogenic RAS and the p53 tumor suppressor. PLoS Biol. 2008;6(12):2853-68.

25. Rodier F, Coppe JP, Patil CK, Hoeijmakers WA, Munoz DP, Raza SR, Freund A, Campeau E, Davalos AR, Campisi J. Persistent DNA damage signalling triggers senescence-associated inflammatory cytokine 
secretion. Nat Cell Biol. 2009;11(8):973-9.

26. Buford TW, Cooke MB, Shelmadine BD, Hudson GM, Redd LL, Willoughby DS. Differential gene expression of Fox01, ID1, and ID3 between young and older men and associations with muscle mass and function. Aging Clin Exp Res. 2011;23(3):170-4.

27. Chien Y, Scuoppo C, Wang X, Fang X, Balgley B, Bolden JE, Premsrirut P, Luo W, Chicas A, Lee CS, et al. Control of the senescence-associated secretory phenotype by NF-kappaB promotes senescence and enhances chemosensitivity. Genes Dev. 2011;25(20):2125-36.

28. Gitenay D, Wiel C, Lallet-Daher H, Vindrieux D, Aubert S, Payen L, Simonnet H, Bernard D. Glucose metabolism and hexosamine pathway regulate oncogene-induced senescence. Cell Death Dis. 2014;5:e1089.

29. Gonin-Giraud S, Mathieu AL, Diocou S, Tomkowiak M, Delorme G, Marvel J. Decreased glycolytic metabolism contributes to but is not the inducer of apoptosis following IL-3-starvation. Cell Death Differ. 2002;9(10):1147-57.

30. James TN. Anatomy of the coronary arteries in health and disease. Circulation. 1965;32(6):1020-33.

31. Jiang P, Du W, Mancuso A, Wellen KE, Yang X. Reciprocal regulation of p53 and malic enzymes modulates metabolism and senescence. Nature. 2013;493(7434):689-93.

32. Quijano C, Cao L, Fergusson MM, Romero H, Liu J, Gutkind S, Rovira II, Mohney RP, Karoly ED, Finkel T. Oncogene-induced senescence results in marked metabolic and bioenergetic alterations. Cell Cycle. 2012;11(7):1383-92.

33. Acin-Perez R, Salazar E, Kamenetsky M, Buck J, Levin LR, Manfredi G. Cyclic AMP produced inside mitochondria regulates oxidative phosphorylation. Cell Metab. 2009;9(3):265-76.

34. Sapio L, Gallo M, Illiano M, Chiosi E, Naviglio D, Spina A, Naviglio S. The Natural cAMP Elevating Compound Forskolin in Cancer Therapy: Is It Time? J Cell Physiol. 2017;232(5):922-7.

35. Gao Y, Raj JU. SQ22536 and W-7 inhibit forskolin-induced cAMP elevation but not relaxation in newborn ovine pulmonary veins. Eur J Pharmacol. 2001;418(1-2):111-6.

36. Bogacka I, Ukropcova B, McNeil M, Gimble JM, Smith SR. Structural and functional consequences of mitochondrial biogenesis in human adipocytes in vitro. J Clin Endocrinol Metab. 2005;90(12):66506.

37. Gonzalez-Sanchez R, Trujillo X, Trujillo-Hernandez B, Vasquez C, Huerta M, Elizalde A. Forskolin versus sodium cromoglycate for prevention of asthma attacks: a single-blinded clinical trial. J Int Med Res. 2006;34(2):200-7.

38. Huerta M, Urzua Z, Trujillo X, Gonzalez-Sanchez R, Trujillo-Hernandez B. Forskolin compared with beclomethasone for prevention of asthma attacks: a single-blind clinical trial. $\mathrm{J}$ Int Med Res. 2010;38(2):661-8.

39. Pateraki I, Andersen-Ranberg J, Hamberger B, Heskes AM, Martens HJ, Zerbe P, Bach SS, Moller BL, Bohlmann J, Hamberger B. Manoyl oxide (13R), the biosynthetic precursor of forskolin, is synthesized in specialized root cork cells in Coleus forskohlii. Plant Physiol. 2014;164(3):1222-36. 
Figures
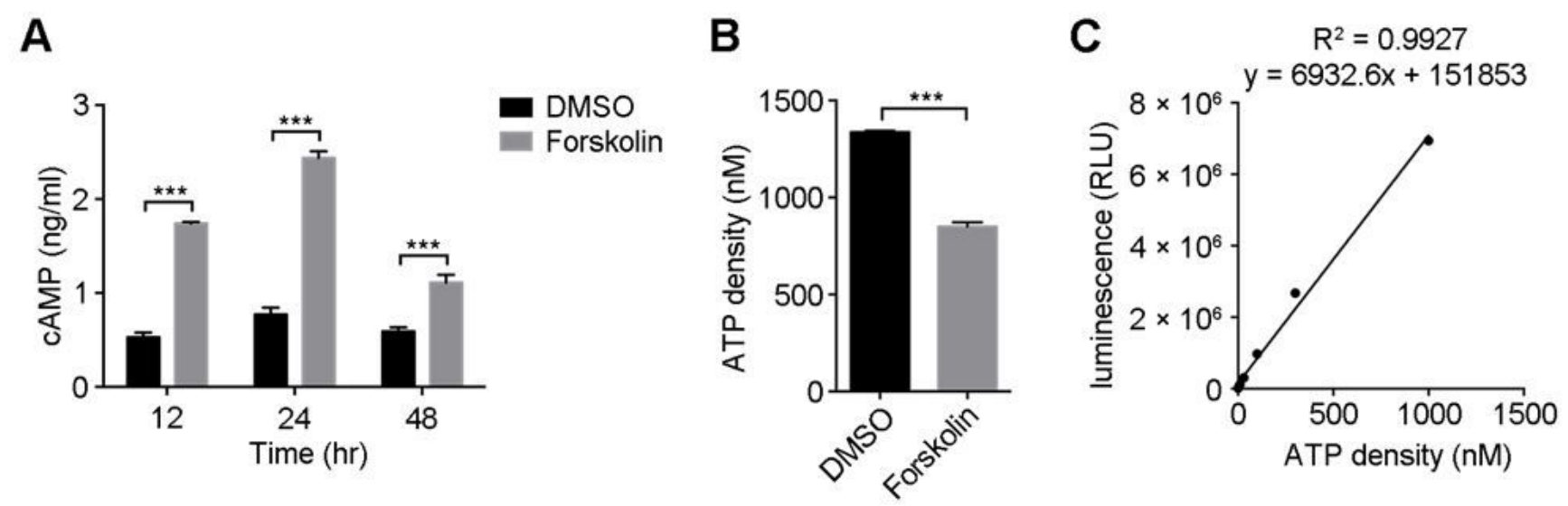

Figure 1

Increase in cAMP level and decrease in ATP level in forskolin-treated MSCs. a Intracellular cAMP levels of MSCs treated with forskolin for 12, 24, or $48 \mathrm{~h}$. b Intracellular ATP content was quantified with an ATP assay kit (left), and a fresh standard curve was prepared (right). Mean \pm SD values are presented in the graph; Student's t-test, $\star \star \star P<0.001$. 


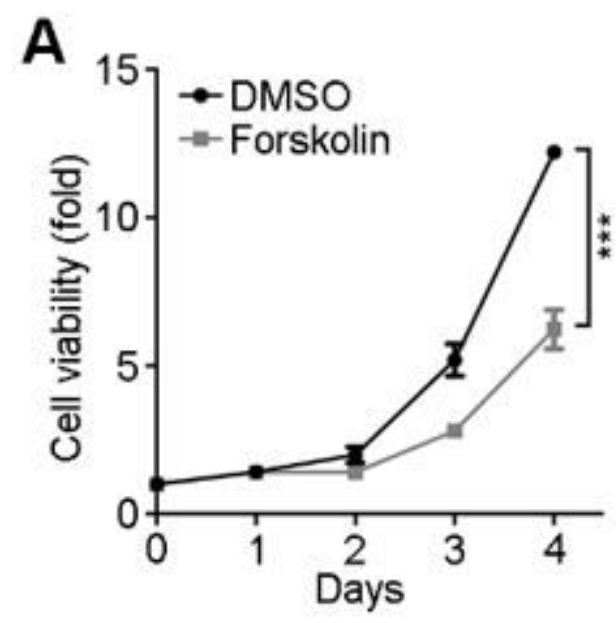

C



B

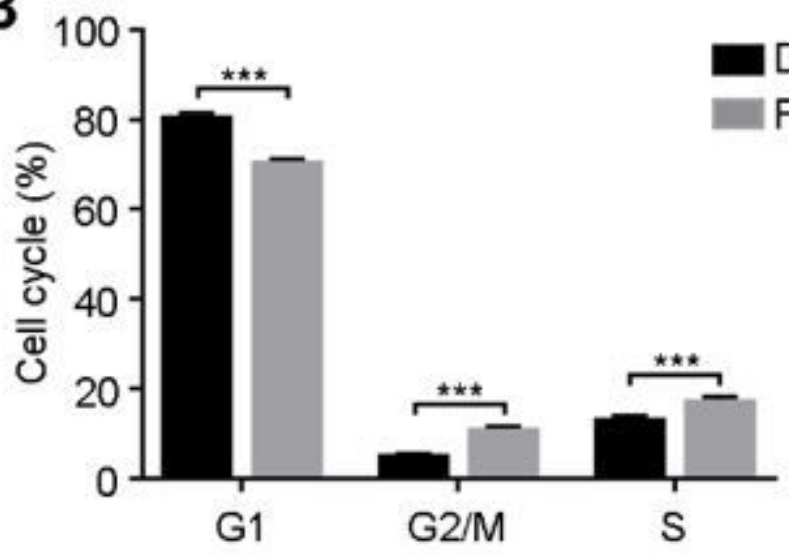

Forskolin
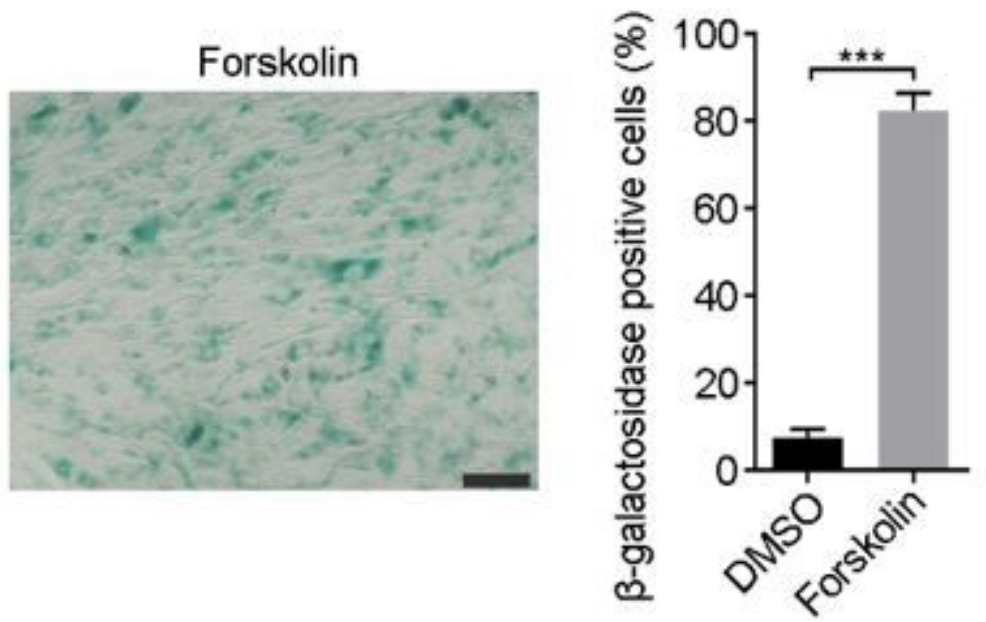

DMSO

Forskolin
D

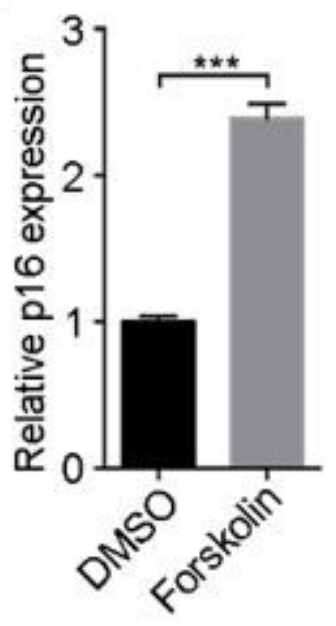

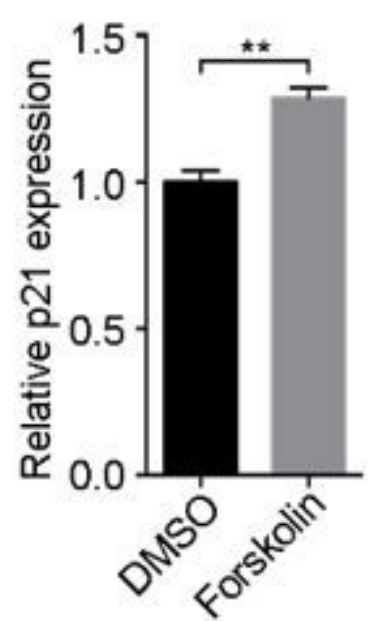

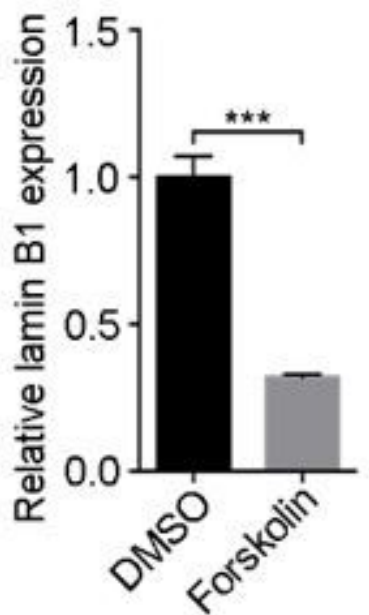

E

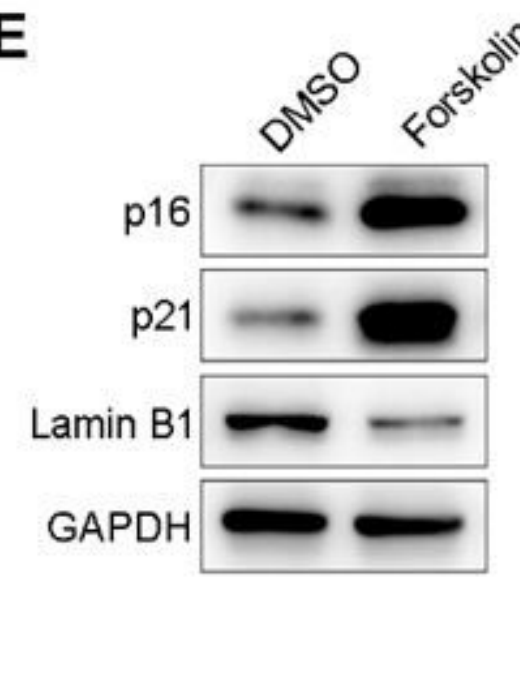

Figure 2

Forskolin causes a senescence phenotype in MSCs. a Relative cell viability of MSCs with or without $24 \mathrm{~h}$ of treatment with forskolin. b Cell-cycle analysis of forskolin-treated and control MSCs. c $\beta$-galactosidase staining of control and forskolin-treated MSCs. d, e Expression of P16, P21, and lamin B1 as assessed with qPCR $d$, and western blotting e of extracts of control and forskolin-treated cells. Mean \pm SD values are presented in the graph; Student's t-test, ${ }^{*} \mathrm{P}<0.05,{ }^{*} \mathrm{P}<0.01,{ }^{*} * \mathrm{P}<0.001$. 
A
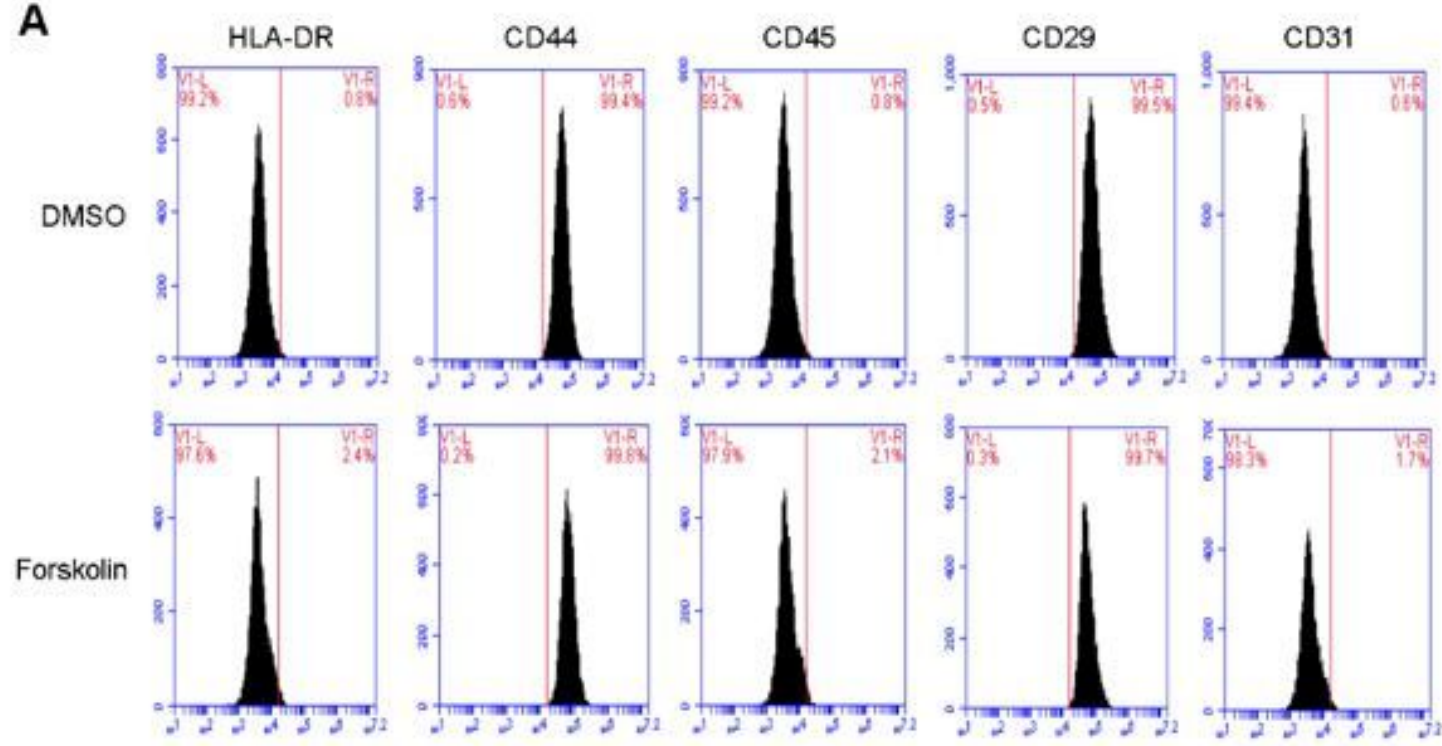

B

DMSO


C

DMSO

Forskolin
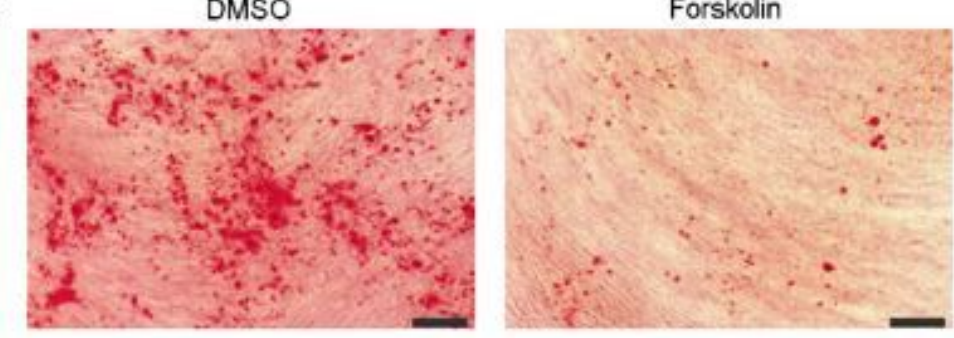

D $=$ Forskolin
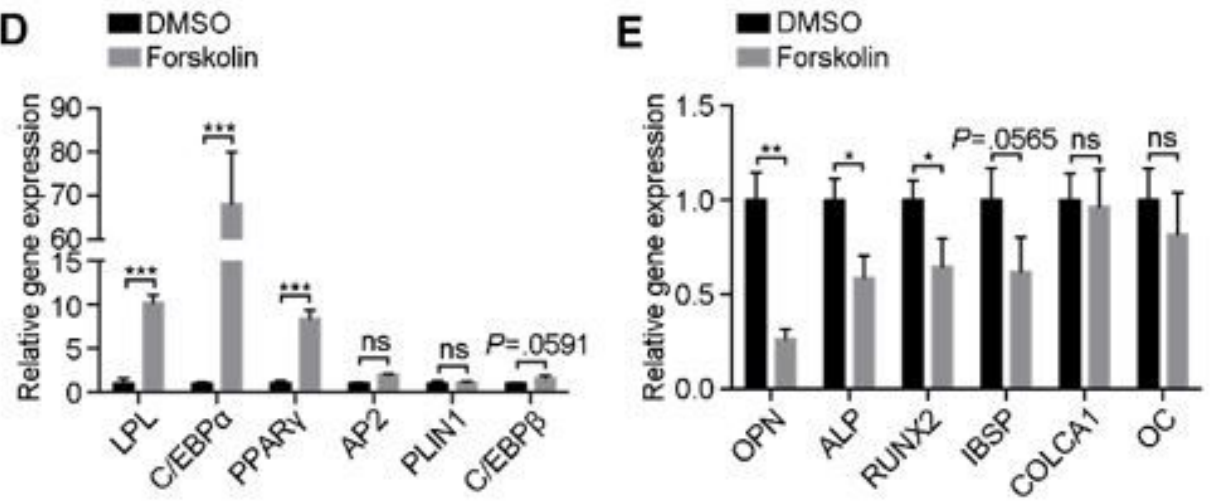

\section{Figure 3}

Immunophenotype and differentiation abilities of forskolin-treated senescent MSCs. a immunophenotyping analysis of control and forskolin-treated MSCs. b Oil red 0 staining for adipocytes (left) and ALP staining for osteoblasts (right) in control or forskolin-treated MSCs. c, d Expression of adipogenesis genes $c$ and osteogenesis genes $d$ as assessed with qPCR. Mean \pm SD values are presented in the graph; Student's t-test, ${ }^{\star} \mathrm{P}<0.05,{ }^{*} \mathrm{P}<0.01,{ }^{* \star *} \mathrm{P}<0.001$. Scale bar, $200 \mu \mathrm{m}$. 
A

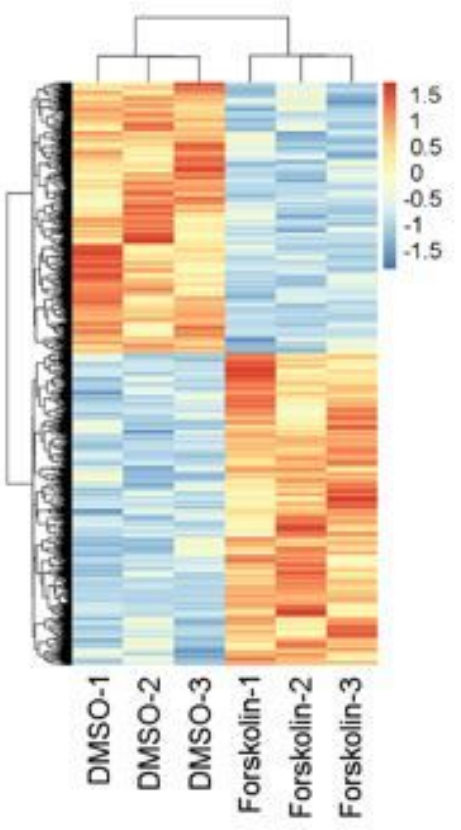

D

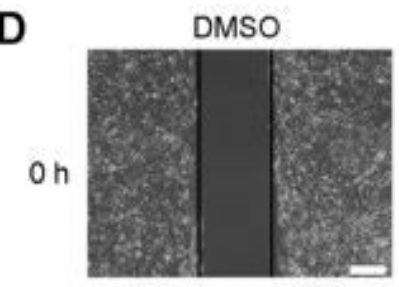

$24 \mathrm{~h}$

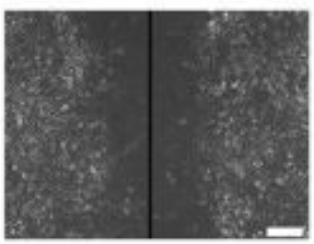

$\mathbf{F}$

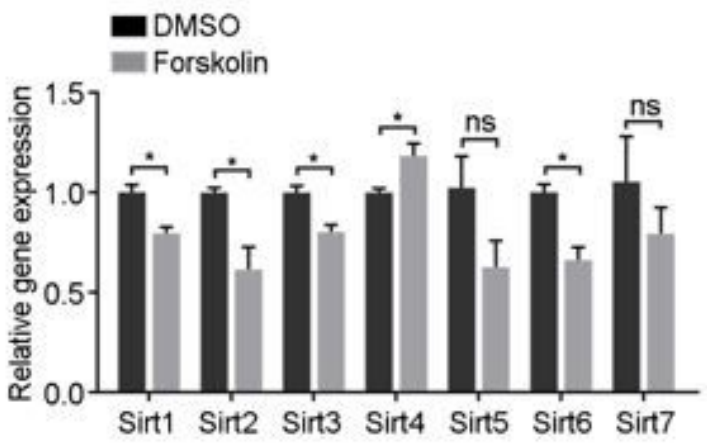

C

Forskolin
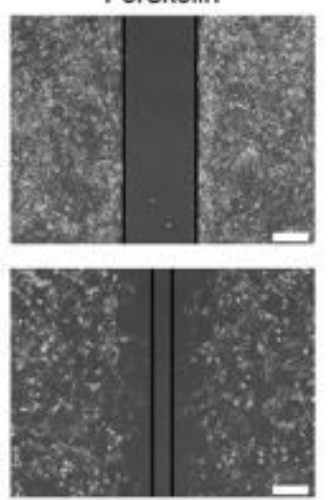

B
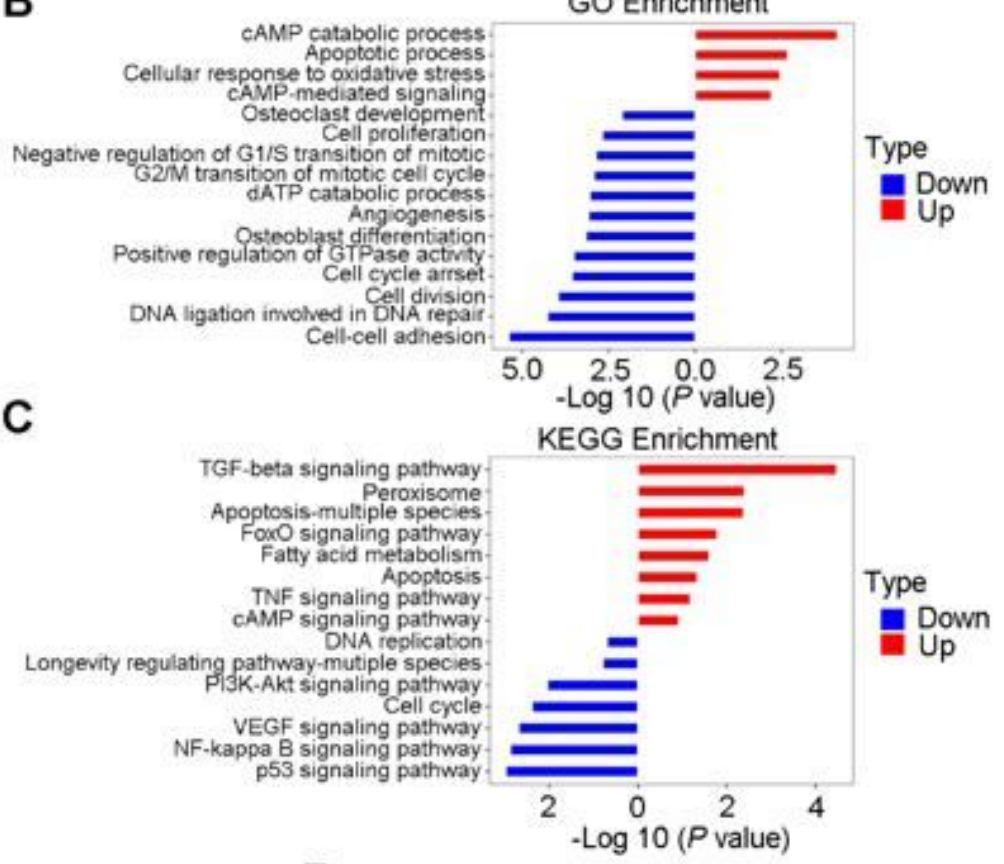

$\mathbf{E}$

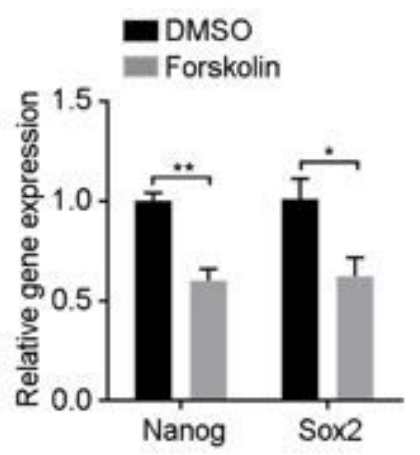

G DMSO

Forskolin

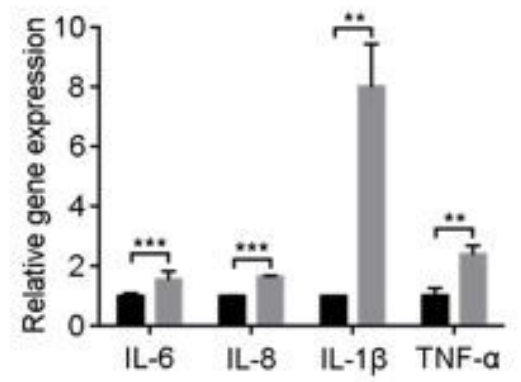

\section{Figure 4}

Cellular functions of senescent MSCs. a Heat map showing differentially expressed genes in control and forskolin-treated MSCs. b, c Top 15 pathways enriched among the Gene Ontology pathways b and KEGG pathways $\mathrm{c}$ with differentially expressed genes between forskolin-treated and non-treated control cells. $\mathrm{d}$ Representative images of wound healing assays using control and forskolin-treated MSCs at time 0 and after $12 \mathrm{~h}$ and $24 \mathrm{~h}$. e Expression of the pluripotent genes encoding Nanog and Sox 2 in control and 
forskolin-treated cells as assessed with qPCR. f Expression of the longevity genes encoding SIRT1-7 in control and forskolin-treated cells as assessed with qPCR. $g$ Expression of the proinflammatory factors IL-6, IL-8, IL-1 $\beta$, and TNF- $\alpha$ in control and forskolin-treated cells as assessed with qPCR. Mean \pm SD values are presented in the graph; Student's t-test, ${ }^{*} \mathrm{P}<0.05,{ }^{*} \mathrm{P}<0.01$, ${ }^{* *} \mathrm{P}<0.001$. Scale bar, $100 \mu \mathrm{m}$.

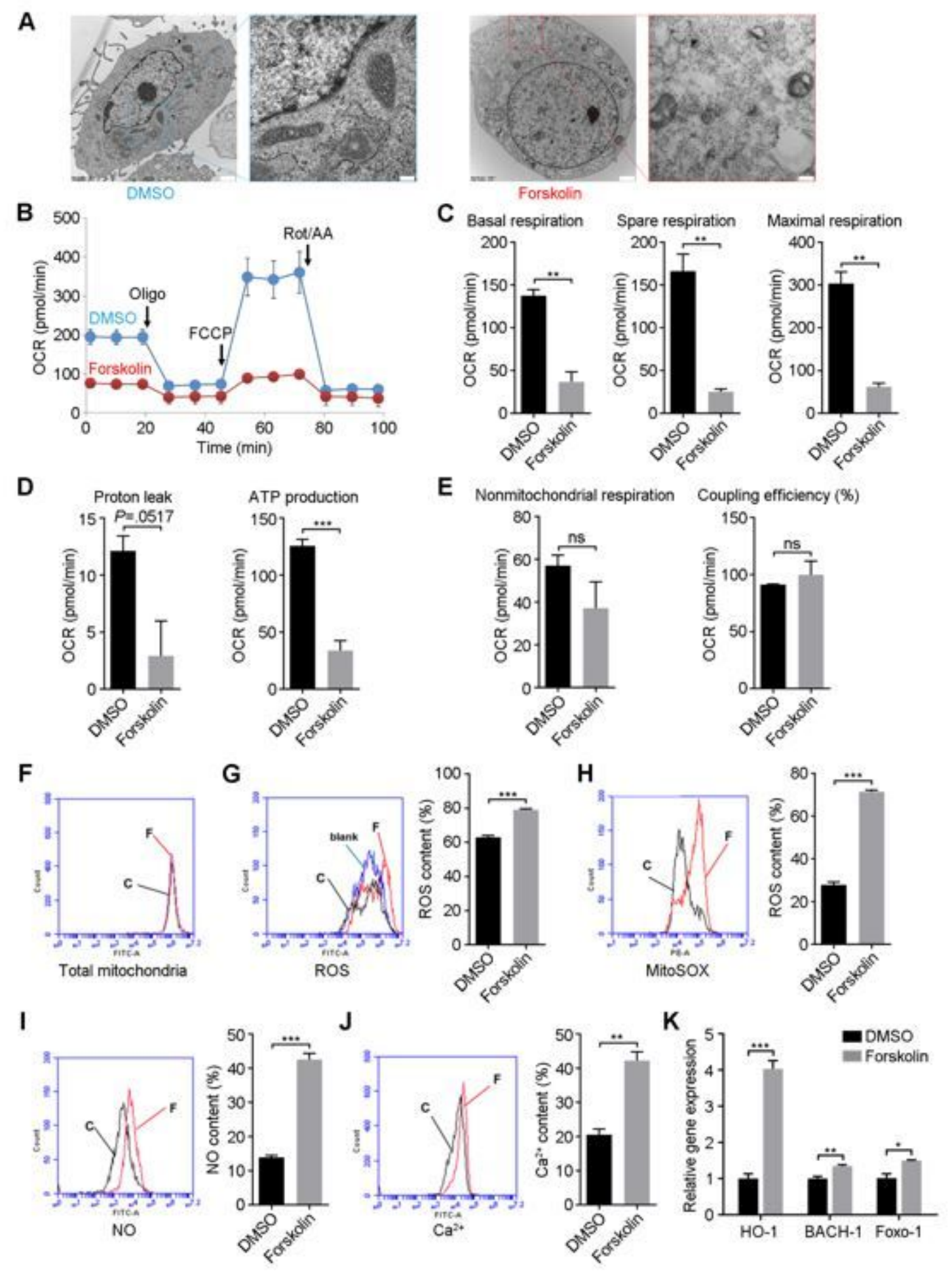

Figure 5 
Analysis of mitochondrial metabolism in senescent MSCs. a Representative transmission electron microscopy images of control and forskolin-treated MSCs. b-e Assessment of mitochondrial respiration metabolism with the Seahorse XF cell Mito Stress test. $f$ Total number of mitochondria stained by MitoTracker Red and examined by flow cytometry. g ROS generation in control and forskolin-treated MSCs. $\mathrm{h}$ Oxidative respiration in mitochondria as assessed by MitoSOX Red mitochondrial SOX indicator staining. i Changes in intracellular NO generation analyzed by DAF-FM diacetate in the NO indication test. j Ca2+ flux analyzed by Fluo-3 AM staining. $\mathrm{k}$ Expression of $\mathrm{HO}-1, \mathrm{BACH}-1$, and $\mathrm{FOXO}-1$ as assessed with qPCR. Mean \pm SD values are presented in the graph; Student's t-test, ${ }^{\star} P<0.05,{ }^{\star} * P<0.01,{ }^{\star} * * P<0.001$, ns: not significant. Scale bars left $=1 \mu \mathrm{m}$, right $=220 \mathrm{~nm}$.

A
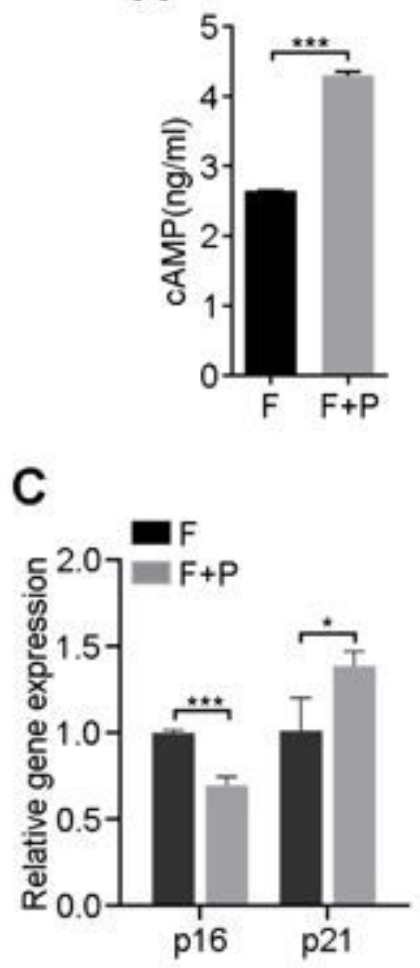

B

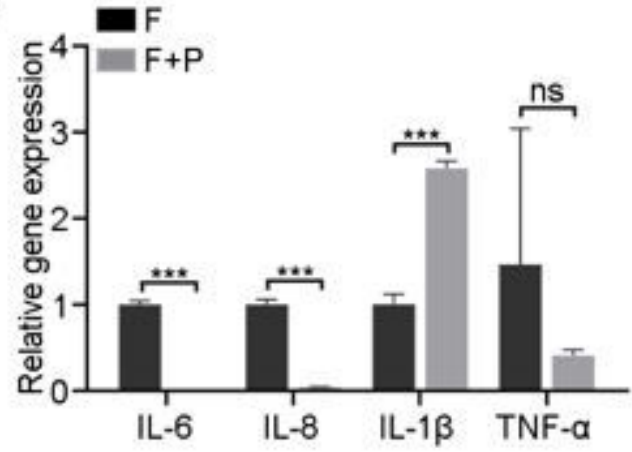

D

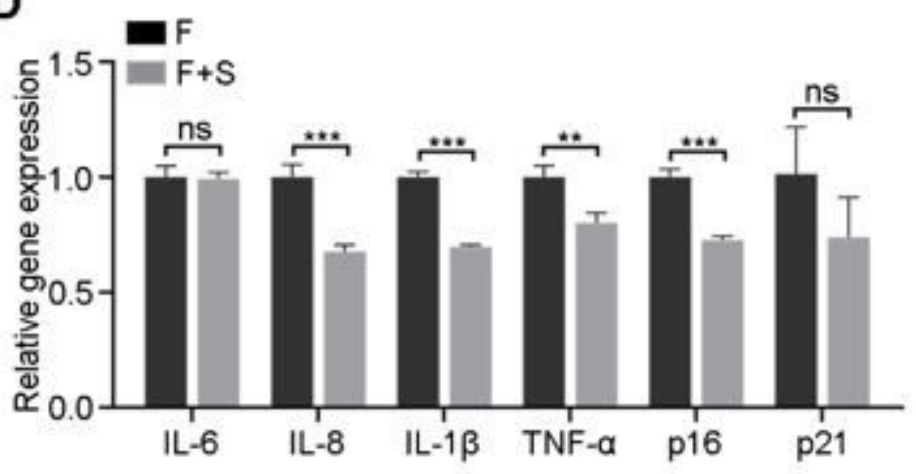

Figure 6

Effects of an NF-KB inhibitor and a cAMP inhibitor on forskolin-induced senescence in MSCs. a cAMP content analysis in the PDTC $(P)+$ forskolin $(F)$ group and the F group. b, $c$ Expression of inflammationrelated genes $b$ and $P 16$ and $P 21 c$ in the $P+F$ and $F$ groups as assessed with qPCR. $d$ Expression of inflammation-related genes and senescence-related genes in the F + SQ22536 (S) group and the F group as assessed with qPCR. PDTC, Pyrrolidine dithiocarbamic acid. Mean \pm SD values are presented in the graph; Student's t-test, ${ }^{*} \mathrm{P}<0.05,{ }^{\star *} \mathrm{P}<0.01,{ }^{\star \star *} \mathrm{P}<0.001$. 


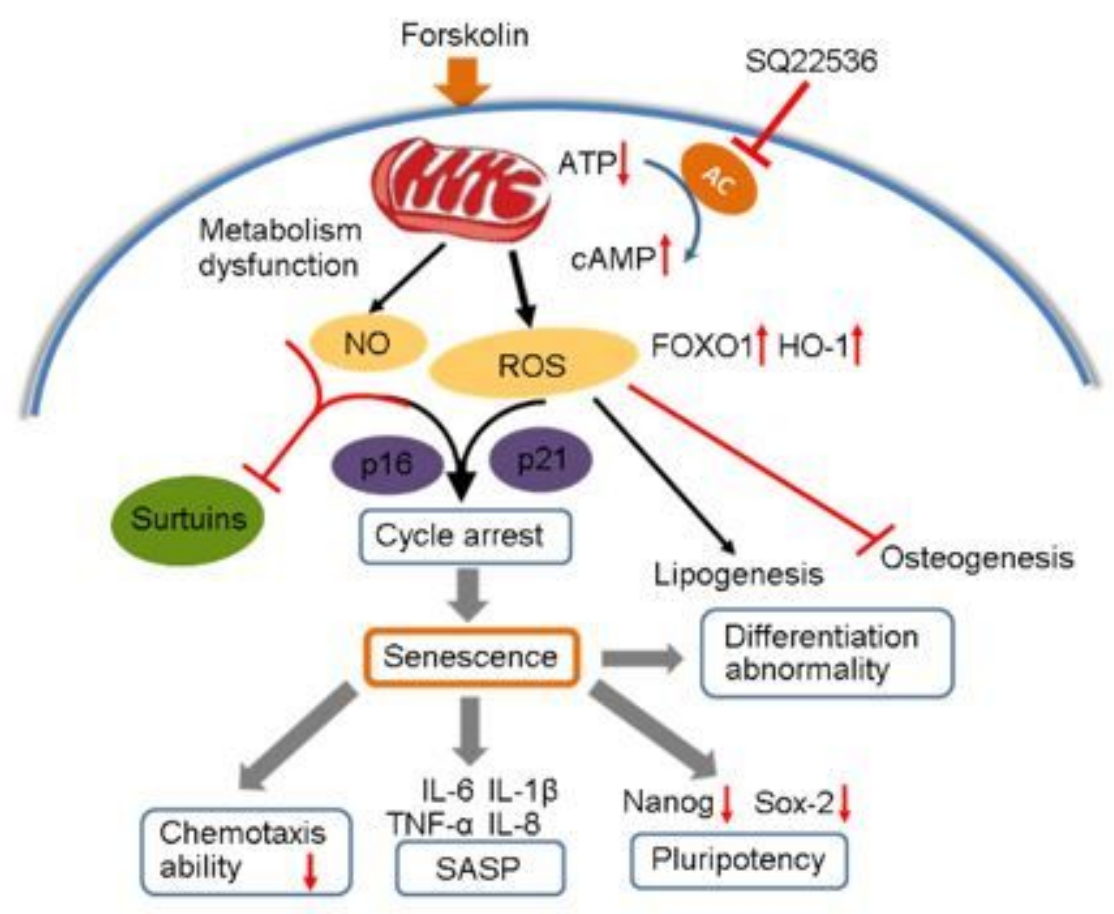

Figure 7

Schematic diagram of the mechanism by which forskolin induces senescence in MSCs. Forskolin induces an increase in CAMP level and a decrease in ATP level, leading to dysfunction of mitochondrial metabolism in MSCs and an increase in the levels of ROS, NO, FOXO1, and HO-1. Additionally, expression of longevity genes sirtuins is suppressed. These changes lead to a senescence phenotype in MSCs characterized by cell-cycle arrest, a SASP, reduced chemotaxis, and abnormalities in pluripotency and differentiation.

\section{Supplementary Files}

This is a list of supplementary files associated with this preprint. Click to download.

- Table1.docx 